\title{
Converting the existing disease surveillance from a paper- based to an electronic-based system using District Health Information System (DHIS-2) for real-time information: The Lebanese experience
}

dalal Ali youssef ( $\square$ dalalyoussef.esu@gmail.com )

\section{Research article}

Keywords: Disease surveillance, District Health Information System, DHIS-2, paper-based, electronic system, Lebanon

Posted Date: November 3rd, 2021

DOI: https://doi.org/10.21203/rs.3.rs-1043203/v1

License: (1) This work is licensed under a Creative Commons Attribution 4.0 International License. Read Full License 


\section{Abstract}

Introduction:

The Ministry of Public Health in Lebanon is in the process of converting the surveillance reporting from a cumbersome paperbased system to a web-based electronic platform (DHIS-2) to have real-time information for early detection of alerts and outbreaks and for initiating a prompt response.

Objectives:

This paper aimed to document the Lebanese experience in implementing DHIS-2 for the disease surveillance system. It also targets to assess the improvement of reporting rates and timeliness of the reported data and to disclose the encountered challenges and opportunities.

Methodology

This is a retrospective description of processes involved in the implementation of the DHIS-2 tool in Lebanon. Initially, it was piloted for the school-based surveillance in 2014; then its use was extended in May 2017 to cover other specific surveillance systems. This included all surveillance programs collecting aggregate data from hospitals, medical centers, dispensaries, or laboratories at the first stage. As part of the national roll-out process, the online application was developed. The customized aggregated-based datasets, organization units, user accounts, specific and generic dashboards were generated. More than 80 training sessions were conducted throughout the country targeting 1290 end-users including health officers at the national and provincial levels, focal persons working in all public and private hospitals, laboratories, and medical centers as well. Completeness and timeliness of reported data were compared before and after the implementation of DHIS-2. Challenges and lessons learned during the roll-out process are listed.

Results

For laboratory-based surveillance, completeness of reporting increased from $70.8 \%$ in May to $89.6 \%$ in October. Timeliness has improved from $25 \%$ to $74 \%$. For medical centers, an improvement of $8.1 \%$ for completeness and $9.4 \%$ in timeliness was recorded before and after training sessions. For zero reporting, completeness remains the same (88\%) and timeliness has improved from $74 \%$ to $87 \%$. The main challenges faced during the implementation of DHIS-2 were mainly infrastructural and system-related in addition to poor internet connectivity and limited workforce and frequent changes to DHIS-2 versions.

Conclusion

Implementation of DHIS-2 improved timeliness and completeness for aggregated data reporting. Continued on-site support, monitoring, and system enhancement are needed to improve the performance of DHIS-2.

\section{Introduction}

The public health surveillance system represents the keystone of public health practices. It was defined as the "continuous, systematic collection, analysis and interpretation of health-related data needed for the planning, implementation, and evaluation of public health practice"(1). Given the importance of timely and accurate information for early detection of alerts and outbreaks as well as for a prompt and adequate response, a real-time consistent and coherent reporting process is crucial for an effective disease surveillance system (2). Of note, reliable data allows public health officials to describe and accurately assess the state of health problems; hence it helps policymakers and investors to effectively allocate resources $(3-5)$.

Given the instrumental role of data collection in any surveillance system (4) in addition to the importance of the used modality for data collection for obtaining reliable data, substantial efforts were exerted during the past few decades for improving data collection such as moving from paper-based surveillance records towards computerized electronic methods $(6,7)$. 
In developed countries, electronic methods are used frequently for data collection, due to their advantages over the paperbased methods (8), by mitigating issues associated with paper-based methods such as avoiding processing duplicate, easing data, better completeness and timeliness, higher quality of data and greater users' acceptance (9-11). However, several challenges related to the collection, aggregation, compilation, analysis, and reporting of health data were encountered in developing countries $(12,13)$. Moreover, limited financial and human resources, lack of sufficient training as well as the use of the traditional paper-based methods in data collection, affected the reliability and timeliness of the data (14-16) which in turn delayed the required outbreak response. Several studies highlighted various problems associated with the traditional paper-based methods use. These snags included the frequent errors occurrence, the low completeness rate, the duplication issues, and the storage costs as well $(17,18)$. Therefore, many developing countries were focusing currently on moving their reporting systems from paper-based methods to electronic methods (19-21)

Among the web-based systems used in developing countries, the District Health information system (DHIS-2) was one of the most popular tools. This free and open-source platform open was developed by the Health Information Systems Program (HISP) at Oslo University. Used by multiple organizations (22), it served as a "tool for collection, validation, analysis, and presentation of aggregate statistical data, tailored but not limited to integrated health information management activities (23, 24). Thus, it facilitates analysis of collected data across all the public health facilities that enhances forecasting of required services for future planning purposes and evaluating the performance of healthcare workers (17). Since its release in 2006, DHIS-2 was used by non-governmental organizations (NGOs) and national governments in more than 60 countries where it was deployed for health-related projects, including monitoring of patient health, improving disease surveillance, pinpointing outbreaks, and speeding up health data access (25).

In Lebanon, the surveillance system has been reactivated after the civil war in 1995, by the ministry of public health to monitor infectious diseases across the country (26). Since then, most cases are reported via fax from different data sources to the epidemiological surveillance unit. In respect of hierarchy, data flow should pass by several steps before reaching the national level which resulted in a delay in receiving information and thus in outbreak detection and response. Automated reporting from data sources directly has been suggested as means to improve the quality of data and timeliness of disease notification and response (27). In 2016, the assessments of the surveillance system conducted in Lebanon (Early warning system EWARS mission and joint external evaluation JEEE) highlighted the importance of moving from a paper-based system and recommended the deployment of a web-based reporting system able to provide real-time information (28). Such migration process required a web-based reporting system using a standard tool compatible with various devices (portable computers, tablets, iPhones, and android), linked with dashboards and alert systems, and supported by international academia. In this context, the DHIS-2 tool was selected to fulfill needed functions. In 2017, the Ministry of Health in Lebanon started the process of migrating surveillance reporting from a cumbersome paper-based system to a web-based electronic platform (DHIS-2). This project aimed to provide real-time information to timely detect alerts and outbreaks. It is of great interest to document the Lebanese experience in implementing the DHIS-2 for diseases surveillance during this transitory phase and to highlight the opportunities and the challenges encountered during this experience. Such information could help and orient the future steps such as the use of DHIS-2 for case-based surveillance.

Therefore, this paper aimed to document the Lebanese experience in implementing DHIS-2 for the disease surveillance system. It also targets to assess the reported data completeness and timeliness improvement and to disclose the encountered challenges and opportunities.

\section{Methods}

This is a retrospective description of processes involved in the implementation of the DHIS-2 tool in Lebanon.

\section{Pilot phase:}


Before initiating the national system roll-out, the DHIS-2 tool was initially piloted in Lebanon in 2014 for school-based absenteeism monitoring surveillance using dedicated national servers. Testing of the usability of this tool was performed to evaluate the potential users' acceptance of the system allowing incorporating of adjustments promptly. Targeted end users were epidemiological surveillance staff. They would be the backbone for DHIS2 implementation. They were requested to provide their feedback in regards to their data entry experience including their insights about the designs of data entry forms, the flexibility, and the data entry easiness as well as the online data entry feasibility.

\section{DHIS-2 customization and setup:}

Before the full-scale implementation of DHIS-2, there was a need to customize the tool to suit the surveillance needs. First, we define data elements that are considered the most important building block of a DHIS-2 database. Data elements represented the "what dimension" to be collected or analyzed. A dictionary of data elements for each reporting form was created.

Aggregated-based datasets were customized according to the format of each paper reporting form. The customized data sets were: Zero reporting form, medical centers reporting form, and laboratory reporting forms. All these forms were reported weekly from different sources: hospitals, medical centers, and laboratories. Additionally, organization units hierarchy was defined and 5 levels were defined as follows: national (level 1), provincial (level 2), district (level 3), locality (level 4), and facility (level 5). The target data sources were: medical centers, mobile clinics, hospitals, and laboratories. Each facility is an orgunit identified within the organization hierarchy. For each organization unit, users' accounts were created specifying the username, password, and accessibility. Access profile is defined according to the user role. It allows data sources to perform data entry, add, update and analyze their data using their computers or mobile. DHIS2 dashboards were introduced for data visualization on an everyday basis and two types of dashboards were generated: specific and generic dashboards. Each facility had access to its dashboard which is a generic one. MOPH users had access to the generic and specific dashboards. To ensure data entry accuracy, validation rules were created. An error message was received by the data operator when they enter any value that is out of the expected range.

\section{National roll-out and training sessions:}

After the official decision to implement DHIS-2 at the data sources level, 80 training sessions targeting 1290 users were conducted throughout the country including two rounds of training for laboratories, two rounds for medical centers, and one round for hospitals. The training material includes a combination of theoretical lectures and hands-on practice. Trained people included district and provincial health officers, 150 designated focal persons working in all public and private hospitals, 140 focal persons from laboratories, and 800 focal persons working in medical centers and dispensaries. In addition, a user guide was developed in 3 languages (English, French, and Arabic) and distributed to data sources during training. A standardized training curriculum and tools are also needed. Furthermore, soft copies of training manuals were shared with staff during training sessions. Given the different data sources where DHIS-2 was used, the DHIS-2 training curriculum was tailored to the needs of health professionals working at different facilities. Subsequently, a circular issued by the ministry of health requested data sources to start reporting their aggregated data to the epidemiological surveillance program using the DHIS-2 platform.

\section{Data flow:}

Data were entered at data sources using DHIS2, then it became available and accessible timely by users depending on their defined roles. Of note, the information was simultaneously available at different hierarchical levels of the system.

\section{Indicators:}

To assess surveillance reporting improvement, indicators were computed such as completeness and timeliness of data. These 2 indicators were compared for the period before and after the implementation of DHIS-2.

\section{Results}


Our results showed an improvement in both completeness and timeliness of data reporting following the implementation of DHIS-2 in our data sources: laboratories, hospitals, and medical centers.

\section{For laboratory surveillance:}

In 2017, a total of 6626 forms were received from all laboratories working inside the hospitals across Lebanon. On average, 120 forms were received on weekly basis. The annual average completeness rate was $78.8 \%$. Starting week 18 of 2017 , an improvement in the completeness was registered which coincide with the roll-out of DHIS-2 for laboratory-based surveillance.

For the period extended from week1 to week 18 in the year 2017, data was received via fax and was entered by the epidemiological unit staff at the peripheral level using DHIS-2. At week 18, the average completeness of laboratory surveillance was $71.4 \%$, it increased to $74.59 \%$ after the first round of training for end-users and reached $85.9 \%$ after the second round (Figure 1).

Table 1 displayed the completeness of laboratory-based surveillance data, before and after, the implementation of DHIS-2 across all provinces. Four provinces registered an average completeness weekly rate equal to or above $90 \%$ after the second round of training.

The improvement of completeness of laboratory data was detected in all provinces. The highest completeness rate was recorded in Nabatyeh (97.92\%) followed by Bekaa province (96.36\%). A noteworthy improvement was revealed in Akkar (64\%) and Beirut (31.1\%) provinces. Starting with the completeness of $1.18 \%$ increasing to $45.45 \%$ after the first round of raining and reaching completeness of $65.83 \%$ after the second round. Similarly, the completeness reached $63.83 \%$ for Beirut province after the second round (Table 1).

Table 1

Completeness of laboratory surveillance data, before and after, the implementation of DHIS-2 in Lebanon

\begin{tabular}{|llllllllll|}
\hline & Akkar & $\begin{array}{c}\text { Baalbeck- } \\
\text { Hermel }\end{array}$ & Beirut & Bekaa & $\begin{array}{l}\text { Mount- } \\
\text { Lebanon }\end{array}$ & Nabatyeh & North & South & Lebanon \\
\hline Phases & & & & & & & & & \\
\hline $\begin{array}{l}\text { Before the } \\
\text { implementation } \\
\text { of DHIS-2 }\end{array}$ & $1.18 \%$ & $91.70 \%$ & $32.74 \%$ & $98.18 \%$ & $67.88 \%$ & $88.24 \%$ & $70.35 \%$ & $88.85 \%$ & $71.44 \%$ \\
\hline $\begin{array}{l}\text { After 1st round } \\
\text { training }\end{array}$ & $45.45 \%$ & $89.41 \%$ & $40.93 \%$ & $100.00 \%$ & $73.64 \%$ & $90.00 \%$ & $64.02 \%$ & $87.56 \%$ & $74.59 \%$ \\
\hline $\begin{array}{l}\text { After second } \\
\text { round training }\end{array}$ & $65.83 \%$ & $91.70 \%$ & $63.83 \%$ & $96.36 \%$ & $81.00 \%$ & $97.92 \%$ & $89.75 \%$ & $94.95 \%$ & $85.90 \%$ \\
\hline
\end{tabular}

In terms of timeliness, an increase from $26.18 \%$ before to $61.87 \%$ after DHIS-2 use was registered at the national level. This improvement was also shown in all provinces. The highest timeliness rate was recorded in Nabatyeh (82.92\%) followed by Baalbeck-Hermel (76.76\%) and South (75.43\%) (Figure 2).

Table 2 displayed the average weekly completeness (AWC) rate before and after use of DHIS-2, in Lebanon for the year 2017. For the zero reporting system, the completeness decreased slightly from $95.54 \%$ before to $88.79 \%$ after the use of DHIS- 2 but still ranked at a good level. However, a completeness improvement was recorded in Nabatyeh (99.41\%) and South (98.14\%) (Table 2). Timeliness was also improved markedly. It reached $79.79 \%$ after the use of DHIS-2 at the national level with an important improvement in timeliness of zero reporting registered in all provinces (Figure 3)

For medical centers: An improvement in the completeness and timeliness was recorded at national and provincial levels (Figure 4). At the national level, the completeness rate was $47.08 \%$ before DHIS-2 use; it reached $58.22 \%$ after DHIS-2. Similarly, for the timeliness, an increase from $9.52-45.15 \%$ was recorded (Table 2 ). 
Table 2

Average weekly completeness (AWC) rate before and after use of DHIS-2, Lebanon 2017

\begin{tabular}{|c|c|c|c|}
\hline & & AWC rate Before & AWC rate After \\
\hline \multirow[t]{9}{*}{ Laboratory surveillance } & Lebanon & 71.46 & 82.66 \\
\hline & Akkar & 20.00 & 60.59 \\
\hline & Baalbeck-Hermel & 92.15 & 90.96 \\
\hline & Beirut & 31.97 & 57.75 \\
\hline & Bekaa & 98.28 & 97.43 \\
\hline & Mount Lebanon & 68.00 & 78.94 \\
\hline & Nabatieh & 88.33 & 95.59 \\
\hline & North & 70.37 & 81.98 \\
\hline & South & 88.88 & 92.72 \\
\hline \multirow[t]{9}{*}{ Medical centers } & Lebanon & 47.08 & 58.22 \\
\hline & Akkar & 21.23 & 43.69 \\
\hline & Baalbeck-Hermel & 21.82 & 54.91 \\
\hline & Beirut & 2.59 & 40.71 \\
\hline & Bekaa & 83.66 & 77.65 \\
\hline & Mount Lebanon & 47.15 & 52.29 \\
\hline & Nabatieh & 68.99 & 79.30 \\
\hline & North & 34.45 & 49.94 \\
\hline & South & 58.20 & 68.89 \\
\hline \multirow[t]{9}{*}{ Zero reporting } & Lebanon & 95.54 & 88.79 \\
\hline & Akkar & 97.71 & 92.94 \\
\hline & Baalbeck-Hermel & 91.45 & 91.21 \\
\hline & Beirut & 86.99 & 76.49 \\
\hline & Bekaa & 99.82 & 99.27 \\
\hline & Mount Lebanon & 96.60 & 93.24 \\
\hline & Nabatieh & 97.71 & 99.41 \\
\hline & North & 94.87 & 64.22 \\
\hline & South & 96.84 & 98.14 \\
\hline
\end{tabular}

\section{Discussion}

The present paper aimed to document the Lebanese experience in implementing DHIS-2 for disease surveillance in Lebanon and to address encountered challenges and opportunities. The use of DHIS-2 in surveillance improved timeliness, completeness, and data quality. DHIS-2 reduced bureaucratic delays associated with paper-based use in surveillance. Data is available in all hierarchical levels upon completion of data entry by the data sources. This improvement in data reporting 
could be due to the numerous trainings performed on focal persons, in addition to the regular follow-up performed by epidemiological surveillance peripheral and central teams. A huge effort was exerted by the epidemiological surveillance unit staff to monitor the process and to support data sources in the initial steps of using DHIS-2. They were also ready and available to assist in case needed. Health officers at the district level conducted on-hand training for the focal persons in data sources who faced difficulty in performing data entry. The mandatory quality checks created at different levels have played a significant role in improving data quality where incorrect data was not allowed to be entered. This was due to system selfvalidation at the data entry level which was based on input validation, identifying applicable ranges, and validation rules. In addition, introducing a dashboard makes DHIS2 compatible to use as a monitoring tool. Our findings were in line with the improvement reported by other countries that implemented a web-based data quality intervention. For example, South Africa has witnessed a significant improvement in data completeness from $26 \%$ before to $64 \%$ as well as overall accuracy of data that increased from $37 \%$ at the first data audit to $65 \%(21)$.

Additionally, data entry errors were minimized due to the setting-up of validation rules. Our results were in line with the findings of a study of the impact of a web-based reporting system on the collection of medication error occurrence data in the United States, where Rudman et al. found that the missing or unspecified data from the cause-of-error variable decreased from $18.6 \%$ to $2.1 \%(9)$.

Besides, verification of data entry errors was swifter since it was directly linked with data sources. It is noteworthy, that the DHIS-2 users' accessibility of their data increased their responsibility feelings in terms of data quality. DHIS-2 could be also beneficial in terms of the economy since it helped to reduce the cost associated with paper use. Therefore, DHIS2 represented a dynamic system that has improved the overall accountability of data reporting from data sources.

In terms of medical centers and laboratories, the completeness was notably improved. However, this was not the case of zero reporting where completeness seems to slightly decrease after the use of DHIS-2. This could be due to the restrained users' familiarity with this platform which is usual during this transition phase. In addition, zero reporting forms were previously actively collected and followed by the MOPH staff responsible for a field visit to hospitals. Our results were in line with many studies conducted in Uganda, Kenya, and South Africa which emphasized an improvement of reporting rates and timeliness of data. All these countries have shown an improvement in reporting rates after the adoption of DHIS-2 $(21,29,30)$.

\section{Implementation challenges:}

Several challenges were encountered during the implementation of DHIS-2 in Lebanon. These challenges were mainly related to infrastructure and human resources. First, the proper communication infrastructure, revealed by limited access to internet services and poor internet connectivity particularly in peripheral areas combined with a scarcity of available electronic devices (computers, tablets....) which makes real-time data entry difficult. Since DHIS-2 is a web-based application, information and communications technology (ICT) infrastructure is essential to support the implementation of this application. Therefore, fast and reliable internet connection and sufficient provision of computer hardware are needed.

Furthermore, internet illiteracy and limited skills in using electronic devices among several end-users were identified as barriers for DHIS-2 implementation in Lebanon. This resulted in a lack of motivation and reluctance to use the new electronic system by these end-users. Addressing the reluctance of end-users through education, continuous training, smooth adaptation to technology, and respecting time needed to be familiar with an electronic system are recommended (31).

Other common challenges were related to human resources challenges such as skilled personnel availability. Our findings were in line with a study conducted in Ghana where limited knowledge of the use of the DHIS-2 software hindered the implementation (32). Hence, there is a need to develop and enhance users' skills through regular trainings on DHIS-2. In addition, DHIS-2 success relied on human capacity, a challenge of staff turn-over and redeployments occurred commonly at data sources (medical centers and laboratories) and the newly recruited staff lacked knowledge and trainings. Hence, passwords updates regularly are needed for security reasons. However, errors in data entry continue to occur but less frequently than paper reporting as validation rules were created. Another key challenge in the implementation of DHIS-2 was 
the frequent DHIS2 versions changes. Lastly, security concerns should also be considered. Of note, this shared experience is based on a short duration of DHIS-2 implementation. Some listed challenges could result from limited familiarity with the new information system during the transition phase.

\section{Lessons learned}

Several lessons learned during the implementation phase in Lebanon are listed below and should be taken into consideration:

- The highest level endorsement for DHIS2 implementation at the MOPH which falls under "political leadership" was crucial for the success of the implementation where all data sources were required officially to start using this tool.

- The importance of the planning and financing of DHIS2 implementation for the success and sustainability of such a project. This included a detailed identification of human, financial, infrastructure, and technical needs. A clear definition of role and responsibilities was also highly recommended

- Training of many focal persons in each data source to limit challenges related to work absenteeism of trained personnel and staff turn-over which impede work.

- Allowing users to change regularly their credentials (passwords) for security reasons.

- data ownership: data sources' access given to their results encourage their involvement in the system.

- Ensuring privacy and security of the platform

- Customizing and formatting the dashboard to make it more interactive.

- Strict monitoring and defining the staffs' role are important.

- Establishing a monitoring and evaluation framework to identify DHIS2-related facilitating factors at all steps ranging from input, process, and outcome

- Regular monthly review meetings to compare are made to track improvements in performance through comparison of the previous month, present month, and yearly national targets. This could help to identify any hindrances to achieving targets

- Refresher training should be performed after every update to the software or data reporting forms, to improve end users' skills and efficiency and to ensure that they are comfortable with what is being done. This constitutes a part of the change management.

- Founding a DHIS2 support team that consists of software specialists who could support any additional required customization of DHIS2 to surveillance needs. It is recommended that this team collaborate with the University of Oslo and HISP.

\section{Future directions}

Before expanding the use of DHIS-2 for case-based surveillance, an extensive and comprehensive evaluation of DHIS-2 implementation is recommended. Concerning users' experience with DHIS-2 implementation, there is a lack of a clear deterrents and enablers understanding of the DHIS-2 use. Given that acceptance of the new system by end-users and their satisfaction are pillars for the success of such implementation, there is a need to assess the acceptability of the DHIS-2 system as an innovation in Lebanon and to understand the key determinants of this acceptance. Besides, an assessment of the overall satisfaction of end-users with DHIS-2 is recommended.

\section{Conclusion}

After 6 months of DHIS-2 use in disease surveillance of aggregate-based data, reporting rates and timeliness of reporting of aggregated data were improved. Errors and bureaucratic delays associated with paper-based systems were minimized as well. The data quality was enhanced and the data management was eased by using an accessible data repository. To fully harness the potential of DHIS-2, the identified challenges must be addressed through continued onsite support, monitoring, and system enhancement. 


\section{Declarations}

Ethics approval and consent to participate

The study protocol is performed following the relevant guidelines. Since this paper falls under public health surveillance, it was exempted for ethical approval at the Ministry of Public Health.

Data Availability:

The data supporting study findings are owned by the Epidemiological Surveillance Program in the Lebanese Ministry of Public Health. Restrictions may apply to the availability of these data. However, Data are available from the authors upon reasonable request after getting the permission of the epidemiological surveillance program head coordinator.

Funding:

No funding was received for manuscript development starting from the study design, data collection, analysis, or interpretation.

Consent for publication

Not applicable.

\section{Competing interests}

The authors declare no competing interests.

Acknowledgments:

We are grateful to the World Health Organization, Beirut country office for the financial and technical support accorded to this project. We also want to acknowledge the efforts of all epidemiological staff at peripheral and central levels who were part of the national roll-out of DHIS2 in Lebanon as well as all focal persons working in reporting data sources who contributed to the success of the implementation process.

Authors Information:

1. Dalal Youssef, MSc, MPH, RSM, PhDc, Epidemiological Surveillance Program \& Clinical Trial Program, Ministry of Public Health, Beirut, Lebanon

2. Ayat Yaghi, IT, Epidemiological Surveillance Program, Ministry of Public Health, Beirut, Lebanon

3. Abbass Jouny, IT, Epidemiological Surveillance Program, Ministry of Public Health, Beirut, Lebanon

4. Linda Abou-Abbas, MPH, PHD, Epidemiological surveillance Program, Ministry of Public Health, Beirut, Lebanon

5. Houssam Chammaa, IT, Lebanon Country Office, World Health Organization, Beirut, Lebanon

6. Nada Ghosn, MD, Epidemiological surveillance Program, Ministry of Public Health, Beirut, Lebanon

Contributions

DY and NG conceived the project and the study design. DY, AY, AJ, HC and NG supported the project implementation in terms of customization and training. DY and LAA drafted the manuscript. DY, LAA, and NG contributed to interpretation and revised for critical content. All authors read and approved the final manuscript.

Corresponding author 
Correspondence to Dalal Youssef.

\section{Abbreviations}

DHIS-2

District Health Information system-2

MOPH

Ministry of Public Health

EWARN

Early warning system

JEE

joint external evaluation

HISP

Health Information Systems Program

ICT

Information and Communications Technology

WHO

World Health Organization

NGOs

Non-Governmental Organizations

\section{References}

1. Thacker SB, Berkelman RL. Public health surveillance in the United States. Epidemiologic reviews. 1988;10:164-90.

2. Isere EE, Fatiregun AA, Ajayi IO. An overview of disease surveillance and notification system in Nigeria and the roles of clinicians in disease outbreak prevention and control. Nigerian medical journal : journal of the Nigeria Medical Association. 2015;56(3):161-8.

3. Kindig D, Day P, Fox DM, Gibson M, Knickman J, Lomas J, et al. What new knowledge would help policymakers better balance investments for optimal health outcomes? Health services research. 2003,38(6 Pt 2):1923-37.

4. Soucie JM. Public health surveillance and data collection: general principles and impact on hemophilia care. Hematology (Amsterdam, Netherlands). 2012;17 Suppl 1:S144-6.

5. Institute of Medicine Committee on Assuring the Health of the Public in the 21st C. The Future of the Public's Health in the 21st Century. Washington (DC): National Academies Press (US)

Copyright 2003 by the National Academy of Sciences. All rights reserved.; 2002.

6. Thriemer K, Ley B, Ame S, K Puri M, Hashim R, Yoon Chang N, et al. Replacing paper data collection forms with electronic data entry in the field: Findings from a study of community-acquired bloodstream infections in Pemba, Zanzibar2012. 113 p.

7. Mukasa O, Mushi HP, Maire N, Ross A, de Savigny D. Do surveys with paper and electronic devices differ in quality and cost? Experience from the Rufiji Health and demographic surveillance system in Tanzania. Global Health Action.

2017;10(1):1387984.

8. Salaffi F, Gasparini S, Ciapetti A, Gutierrez M, Grassi W. Usability of an innovative and interactive electronic system for collection of patient-reported data in axial spondyloarthritis: comparison with the traditional paper-administered format. Rheumatology (Oxford, England). 2013;52(11):2062-70. 
9. William J. Rudman, Jessica H. Bailey, Carol Hope, Paula Garrett, Brown CA. The Impact of a Web-based Reporting System on the Collection of Medication Error Occurrence Data Advances in Patient Safety. 2005;3.

10. Wood C, von Baeyer CL, Falinower S, Moyse D, Annequin D, Legout V. Electronic and paper versions of a faces pain intensity scale: concordance and preference in hospitalized children. BMC pediatrics. 2011;11:87.

11. Ma S, Lawpoolsri S. Effectiveness of Implementation of Electronic Malaria Information System as the National Malaria Surveillance System in Thailand. 2016;2(1):e20.

12. AbouZahr C, Boerma T. Health information systems: the foundations of public health. Bulletin of the World Health Organization. 2005;83(8):578-83.

13. MR. O. A review on barriers to implementing health informatics in developing countries. . Journal of Health Informatics in Developing Countries 2007;1(1):19-22.

14. Benson MB. "Adoption of Hospital Information Systems in Nigeria.". Journal of Global Health Care Systems. 2011;1(no.3).

15. Kushniruk, AW, V. L. Patel, Cimino aJJ. "Usability Testing in Medical Informatics: Cognitive Approaches to Evaluation of Information Systems and User Interfaces.”. Proceedings of the AMIA Annual Fall Symposium 1997;:218.

16. Bangor A, Kortum PT, Miller JT. An Empirical Evaluation of the System Usability Scale. International Journal of HumanComputer Interaction. 2008;24(6):574-94.

17. Garrib A, Stoops N, McKenzie A, Dlamini L, Govender T, Rohde J, et al. An evaluation of the District Health Information System in rural South Africa2008. 549-52 p.

18. Makombe S, Hochgesang M, Jahn A, Tweya H, Hedt B, Chuka S, et al. Assessing the quality of data aggregated by antiretroviral treatment clinics in Malawi2008. 310-4 p.

19. Abiy R, Gashu K, Asemaw T, Mitiku M, Fekadie B, Abebaw Z, et al. A Comparison of Electronic Medical Record Data to Paper Records in Antiretroviral Therapy Clinic in Ethiopia: What is affecting the Quality of the Data? Online journal of public health informatics. 2018;10(2):e212.

20. Stausberg J, Koch D, Ingenerf J, Betzler M. Comparing paper-based with electronic patient records: lessons learned during a study on diagnosis and procedure codes. Journal of the American Medical Informatics Association : JAMIA. 2003;10(5):470-7.

21. Mphatswe W, Mate KS, Bennett B, Ngidi H, Reddy J, Barker PM, et al. Improving public health information: a data quality intervention in KwaZulu-Natal, South Africa. Bulletin of the World Health Organization. 2012;90:176-82.

22. El-Ibiary SY, Yam L, Lee KC. Assessment of burnout and associated risk factors among pharmacy practice faculty in the United States. American journal of pharmaceutical education. 2017;81(4).

23. Johnson RE, Ried LD, Robertson N. Self-reported burnout among HMO pharmacists. Journal of pharmaceutical marketing \& management. 1987;2(2):107-27.

24. Braa J, Kanter AS, Lesh N, Crichton R, al. JBe. "Comprehensive yet scalable health information systems for low resource settings: a collaborative effort in Sierra Leone". AMIA Annual Symposium Proceedings-American Medical Informatics Association 2010:372-6.

25. Smyth JM. DHIS2 - Transforming Health IT Standards in the Developing World. Open Health news. 2017. 
26. Ghosn N, Nasredine A, Baddour YM, Coulombier D, Nasserdine S. Electronic surveillance of outbreaks in Lebanon. BMC proceedings. 2008;2 Suppl 3:S2.

27. Nsubuga P, White ME, Thacker SB, Anderson MA, Blount SB, Broome CV, et al. Public Health Surveillance: A Tool for Targeting and Monitoring Interventions. In: nd, Jamison DT, Breman JG, Measham AR, Alleyne G, Claeson M, et al., editors. Disease Control Priorities in Developing Countries. Washington (DC): World Bank The International Bank for Reconstruction and Development/The World Bank Group.; 2006.

28. World Health O. Joint external evaluation of IHR core capacities of the Lebanese Republic: mission report, 25-29 July 2016. Geneva: World Health Organization; 2017 2017. Contract No.: WHO/WHE/CPI/2017.2.

29. Manya A, Braa J, Øverland LH, Titlestad OH, Mumo J, Nzioka C, editors. National roll out of District Health Information Software (DHIS 2) in Kenya, 2011-Central server and Cloud based infrastructure. IST-Africa 2012 conference proceedings; 2012: IIMC International Information Management Corporation.

30. Gammersvik $\varnothing$. Balancing institutional needs with generic functionality in information systems: the biography and evolution of the DHIS 2 tracker following two implementations in Palestine 2015.

31. Khobi JA, Mtebe JS, Mbelwa JT. Factors influencing District Health Information System usage in Sierra Leone: A study using the Technology-Organization-Environment Framework. The Electronic Journal of Information Systems in Developing Countries. 2020;86(6):e12140.

32. Jolliffe B, Poppe O, Adaletey D, Braa J. Models for Online Computing in Developing Countries: Issues and Deliberations. Information Technology for Development. 2015;21(1):151-61.

\section{Figures}

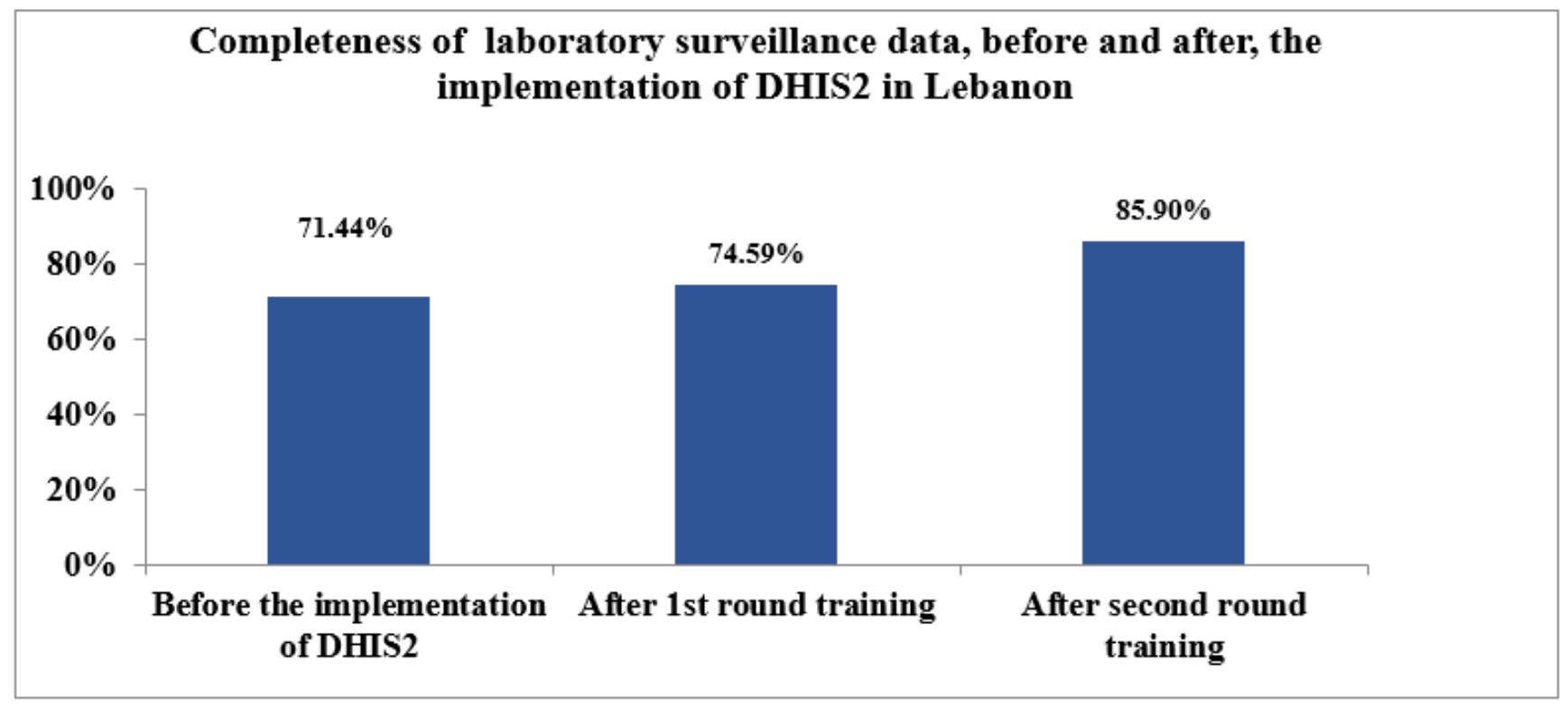

Figure 1

Completeness of laboratory surveillance data before and after the implementation of DHIS2 in Lebanon 


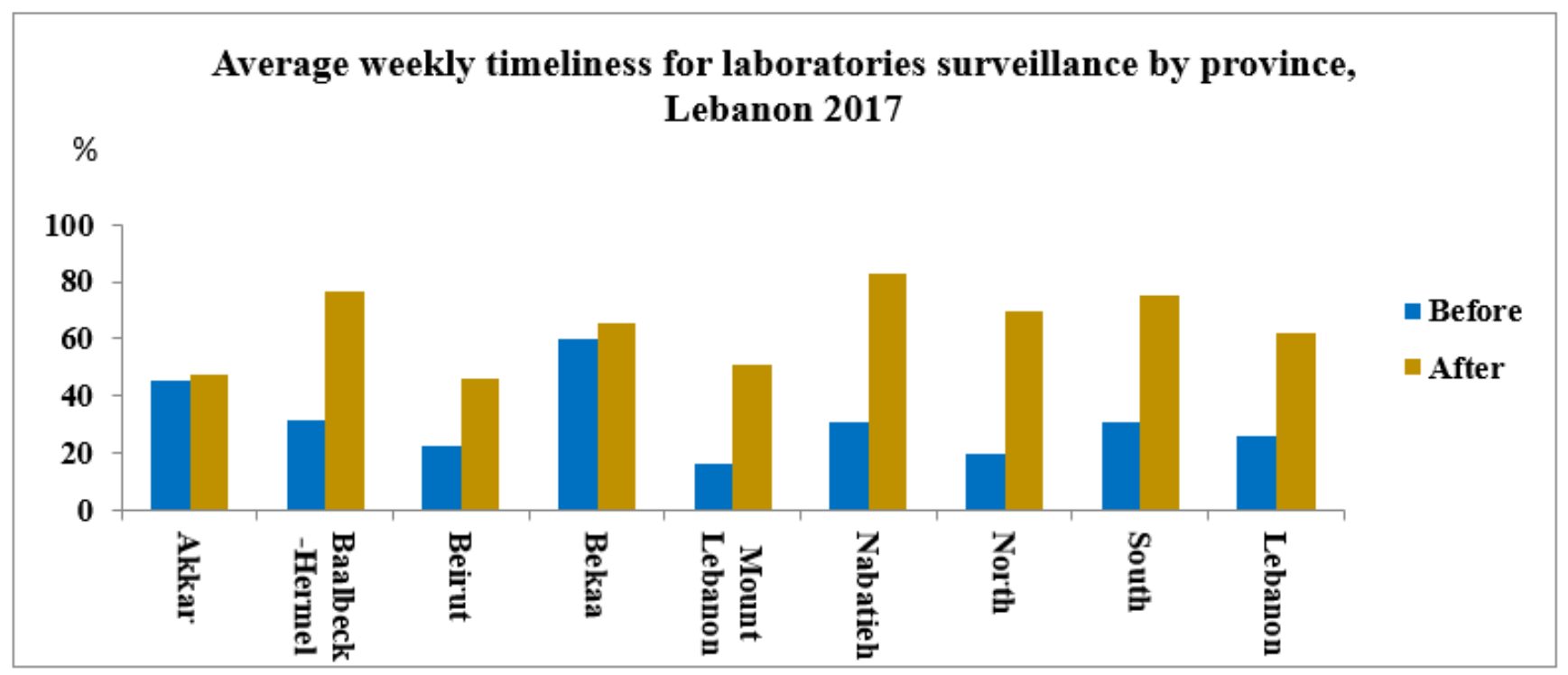

Figure 2

Average weekly timeliness for laboratories surveillance system by province, Lebanon 2017

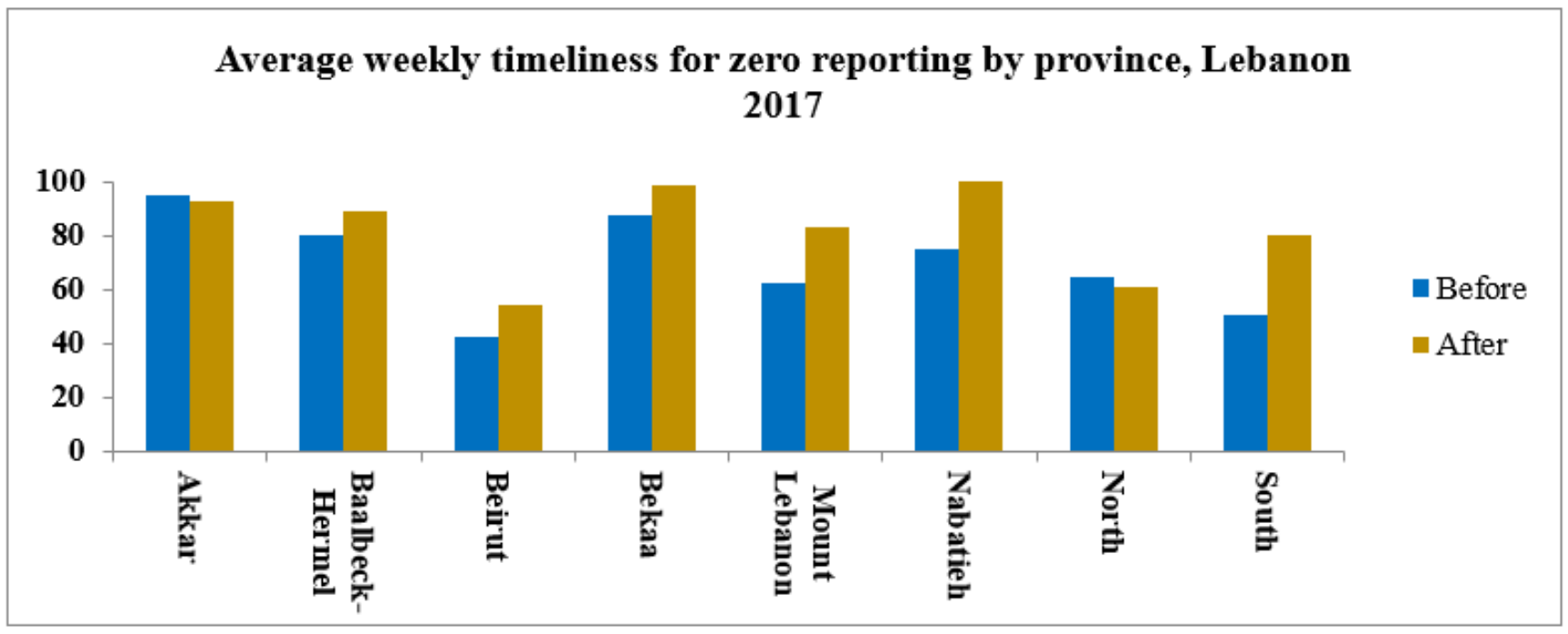

Figure 3

Average weekly timeliness for zero reporting by province, Lebanon 2017 


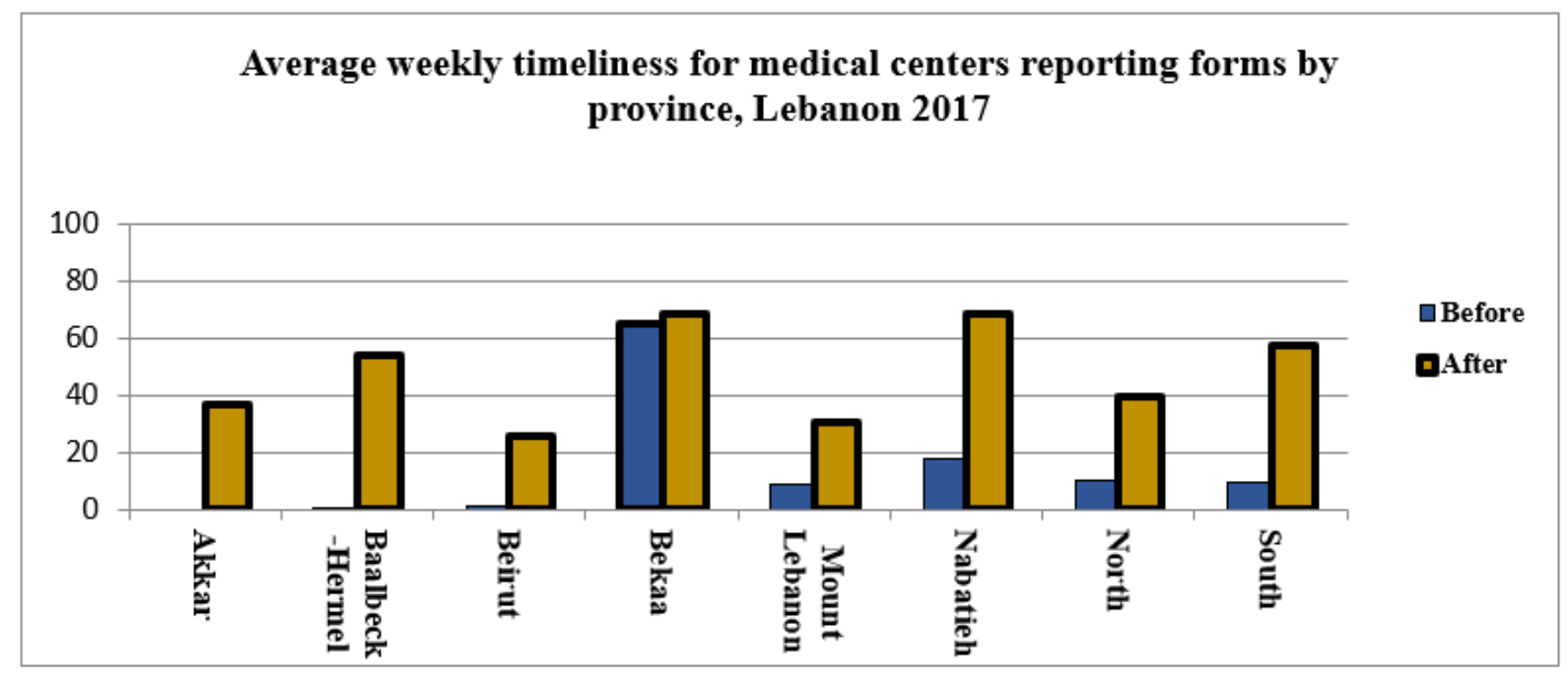

Figure 4

Average weekly timeliness for medical centers reporting forms by province, Lebanon 2017 